\title{
Dynamics of gas hydrate: case of the Congo continental slope
}

\author{
N. Sultan ${ }^{a}$, J. P. Foucher ${ }^{a}$, P. Cochonat ${ }^{a}$, T. Tonnerre ${ }^{a}$, J. F. Bourillet ${ }^{a}$, H. Ondreas ${ }^{a}$, \\ E. Cauquil ${ }^{b}$ and D. Grauls ${ }^{b}$ \\ ${ }^{a}$ Departement de Geosciences Marines, Technopole de Brest-Iroise, Ifremer Brest, B.P. 70, Plouzané F-29280, \\ France \\ ${ }^{\mathrm{b}}$ Total, Paris, France \\ *: Corresponding author : Tel.: +33-02-98-22-42-59; fax: +33-02-98-22-45-70. Nabil.Sultan@ifremer.fr
}

\begin{abstract}
A numerical model is developed to study the temperature effect on stability and phase transformation of gas-hydrate. The model uses a mathematical formulation based on the enthalpy form of the conservation law of energy. The use of the enthalpy form instead of the temperature form as often done in the literature has made the problem numerically simpler. The model is then applied to describe the effect of sea bottom temperature variations on the stability of gas hydrate occurrences and on the seafloor reflectivity in sediments of the Congo continental slope. Indeed, a migrating seafloor reflectivity front is observed on 3D seimic images from two surveys performed 6 months apart and interpreted as a migrating gas hydrate stability zone. Seawater temperature variations were recorded over a 230-day period. At the seafloor, the amplitude of these variations could explain a 75$\mathrm{m}$ shift in water depth of the upper limit of the gas hydrate stability zone. However, the response of the sediment to a perturbation applied at the seafloor is not immediate because of the effects of thermal diffusion and of latent heat of hydrate dissociation. Thermal modelling shows that the depth of penetration of these perturbations is around $2 \mathrm{~m}$ for the saturated sediment and around $6 \mathrm{~m}$ for hydrate-bearing sediments (hydrate fraction of 0.1 ). The switch between gas and gas hydrate generated by these temperature fluctuations concern only the first meter of sediment. These appear too small to explain the migration of the seafloor reflectivity, considering that the wavelength of the seismic signal is significantly larger (around $10 \mathrm{~m}$ ). However, for temperature fluctuations with larger wavelength the switch between gas and gas hydrate will be more important and consequently could explain the reflectivity contrast as a possible migrating gas or gas hydrate front.
\end{abstract}

Keywords: continental margins; finite difference; heat flow; gas-hydrate; seafloor reflectivity; stefan problem; temperature 


\section{Introduction}

Gas (usually methane) in marine soil results from thermogenic or biogenic formation within seabed soils. Depending on the methane concentration and the temperature and pressure conditions, methane occurs in sediment either as a dissolved component, as free gas, or as solid hydrate. For suitable temperature and pressure conditions, methane hydrates may only crystallize from natural gases when gas concentrations exceed the methane solubility in the pore water. In the last two decades, industrial and scientific concerns have initiated numerous studies concerning the dynamic of marine gas hydrate in relation with gas transport, drilling hazards, environmental hazards, global warming and submarine slides. In this paper, a numerical model of solid gas hydrate dissociation on the slope of a continental margin in response to a change of the bottom water temperature is developed. A general application of the model is to estimate melting rates and consequences of the gas release on the physical properties of the slope sediments. Previous studies in this field include the work by Chaouch and Briaud (1997) who investigated gas hydrate melting around a warm oil pipe and Delisle et al (1998) who proposed a model of thermal re-equilibration of slope sediments following a slump. All previous studies have emphasized the important role played by the latent heat of formation and dissociation of gas hydrate in the heat transfer process (see for instance Briaud and Chaouch 1997). Our approach enters into the type of modelling developed by these authors. However, it uses a new formulation for the hydrate stability based on the enthalpy form of the law of conservation of energy. In the second part of the paper, we apply the model to describe the effect of sea bottom temperature changes on the gas hydrate occurrences in sediments of the Congo continental slope and on the seafloor reflectivity.

\section{Numerical models of gas hydrate in marine sediments}

Quantitative dynamic models of gas hydrate in marine sediments can be grouped into two categories. The fundamental solved equations in the first category are those for conservation 
of momentum, fluid mass and energy for transient and steady state regime. A first class of these steady-state models takes into account methane conservation, the supply of gas being then considered (Rempel and Buffett, 1997; 1998; Xu and Ruppel, 1999). Whereas Rempel and Buffett (1997) ignore diffusion in fluid phase, Rempel and Buffett (1998) and Xu and Ruppel (1999) propose a complete treatment of the gas (methane) advective-diffusive flow coupled with heat transfer. Egeberg and Dickens (1999) consider the chloride content of the pore fluid, but their model does not take into account the dissolved methane in the pore fluid. Davie and Buffett (2001) couple both approaches: they simulate the pore fluid chemistry, both in terms of chloride content and dissolved methane in gas and hydrate phases, the methane migration being taken into account either by advection or by diffusion in fluid phase when dissolved. Another line of inquiry deals with models, which are often conceptually simpler: the only solved equation is usually the energy conservation in transient regime. Mienert et al. (2001) work on the modeling of the Hydrate Stability Zone (HSZ) as a function of temperature and pressure. They show a distinct decrease of the HSZ at the Norwegian margin from the last glacial maximum (LGM) to the present time. Briaud and Chaouch (1997) propose a model of gas hydrate dissociation beneath oil platform due to the heat released around pipes where hot oil travels from the well to the platform. They report that melting process generates a large amount of gas that can endanger the stability of the foundation. Delisle et al. (1998) propose a model of gas hydrate formation due to the thermal reequilibration occurring after slumps, actually to gas hydrate formation due to the seafloor cooling. The main result suggests that the structure do not regain complete thermal equilibrium after slumping in the course of several tens of years. Both Briaud and Chaouch (1997) and Delisle et al. (1998) emphasize that the latent heat greatly impedes gas hydrate formation and dissociation: additional heat source and heat sink are produced as gas hydrate 
forms and dissociates respectively. The model we present in this study enters this last type of models where only the energy conservation equation in transient regime is considered.

\section{II.1 Proposed model}

Numerous empirical laws for gas hydrate stability have been derived from theoretical computations and laboratory experimental data. Pressure (or water depth) versus temperature phase diagrams are sensitive to the gas nature (methane, ethane...) (see for instance Sloan 1998), the dissolved ion concentration (Dickens and Quinby-Hunt 1994) and the pore size distribution (see for instance Handa and Stupin 1992 and Henry et al. 1999). In the lack of relevant data concerning the chemical composition of the gas at the study site on the Congo continental slope, we assume that methane and ethane are the only two gas components present within the sediment. In addition, the effect of the capillary pressure generated by the pore radius on the stability law is small in marine sediments (Henry et al. 1999); that is why, only the effect of the salinity of the seawater is considered.

In this work, the stability law for pure methane in seawater in the P-T space is given by the following equation:

$$
T=9.49468 \ln \left(\frac{p}{p_{0}}\right)+263.212
$$

where $\mathrm{T}$ is the temperature in $\mathrm{K}, \mathrm{p}_{0}$ is a reference pressure equal to $1 \mathrm{MPa}$ and $\mathrm{p}$ is the hydrostatic pressure in MPa. This equation is based on the experimental results presented in Dickens and Quinby-Hunt (1994). As shown in Figure 1, equation [1] is valid only for hydrostatic pressures greater than about $3 \mathrm{MPa}$.

The analysis of heat transfer with a moving solid-fluid boundary is often referred to as a Stefan problem. For material undergoing a phase transformation, the conservation of energy is given by the following equation: 


$$
\bar{C}_{p} \frac{\partial T}{\partial t}+\nabla\left(h_{l} \underline{u_{l}}\right)=\nabla(\bar{\kappa} \nabla T)+\phi L \frac{\partial \eta}{\partial t}
$$

where $h_{l}$ is the enthalpy of the liquid phase and is given by:

$$
h_{l}=C p_{l}\left(T-T_{m}\right)+L
$$

The effective volumetric heat capacity of the medium $\bar{C}_{p}$ is expressed by the following expression:

$$
\bar{C}_{p}=C_{p w} \phi(1-\eta)+C_{p h} \phi \eta+C_{p s}(1-\phi)
$$

For equations [2], [3] and [4] the subscript $s$ denotes the solid phase, the subscript $h$ denotes the hydrate phase and the subscript $l$ denotes the liquid phase; $u_{l}$ is the velocity of the liquid phase, $\phi$ is porosity, $\eta$ is the hydrate fraction, $T$ is temperature, $T_{m}$ is the melting temperature and $L$ is the latent heat of fusion. In equation $2, \bar{\kappa}$ is the effective thermal conductivity of the medium. According to 2001 MBARI Gas Hydrate Workshop conclusions, the thermal conductivity of the hydrate-sediment medium is one of the key knowledge gaps in hydrate modelling. The effective thermal conductivity of any porous solid is bounded by the harmonic and geometric mean of the thermal conductivity of the constituents. In this work, the coefficient $\bar{\kappa}$ is expressed by equation 5 (harmonic mean):

$$
\bar{\kappa}=\frac{\kappa_{l} \kappa_{h} \kappa_{s}}{\kappa_{h} \kappa_{s} \phi(1-\eta)+\kappa_{l} \kappa_{s} \phi \eta+\kappa_{l} \kappa_{h}(1-\phi)}
$$

For free gas trapped in the sediment, the thermal conductivity of the hydrate is replaced by the thermal conductivity of the gas $\left(\kappa_{\mathrm{g}}\right)$ and the hydrate fraction by the gas fraction.

In equation 4 , the effect of the gas phase on the volumetric heat capacity is neglected. Indeed,

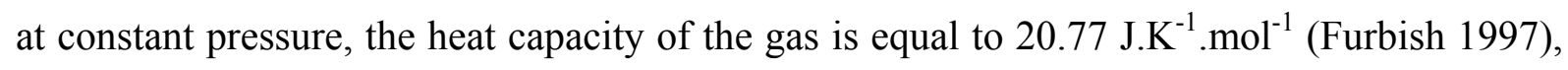
which is around fifty times lower than the heat capacity of the liquid and solid phases. 
In this study, the enthalpy form of the conservation energy is considered in one spatial dimension and flow in the liquid is neglected $\left(u_{l}=0\right)$. Under these assumptions, equation [2] is simplified to:

$$
\bar{C}_{p} \frac{\partial T}{\partial t}=\frac{\partial}{\partial x}\left(\bar{\kappa} \frac{\partial T}{\partial x}\right)+\phi L \frac{\partial \eta}{\partial t}
$$

The complexity to solve equation [6] is the availability of one equation for two unknowns: the temperature $T$ and the hydrate fraction $\eta$.

The conservation of energy of two-phase mixture can be expressed in terms of temperature and total volumetric enthalpy H. Indeed:

$$
\frac{\partial H}{\partial t}=\bar{C}_{p} \frac{\partial T}{\partial t}-\phi L \frac{\partial \eta}{\partial t}
$$

Thus, equation [6] is simplified and the enthalpy form of the conservation of energy is given by the following simplified equation:

$$
\frac{\partial H}{\partial t}=\frac{\partial}{\partial x}\left(\bar{\kappa} \frac{\partial T}{\partial x}\right)
$$

For a pure material, the temperature can be expressed as a function of the enthalpy. For a material with a melting temperature $\mathrm{T}_{\mathrm{m}}, \mathrm{T}(\mathrm{H})$ is defined as (Figure 2):

$$
T(H)=\left\{\begin{array}{lr}
\frac{H}{C p_{s}}+T_{m} & H<0 \\
T_{m} & 0 \leq H \leq L \\
\frac{H-L}{C p_{l}}+T_{m} & H>L
\end{array}\right.
$$

In a porous medium containing gas hydrate, equation [9] becomes:

$$
T(H)=\left\{\begin{array}{lr}
\frac{H}{\overline{C p}}+T_{m}(p) & H<0 \\
T_{m}(p) & 0 \leq H \leq L \\
\frac{H-L}{C p}+T_{m}(p) & H>L
\end{array}\right.
$$


where $p$ is the hydrostatic pressure (Figure 1). In the case where we can express temperature as a function of enthalpy $T=T(H)$, equation [8] becomes:

$$
\frac{\partial H}{\partial t}=\frac{\partial}{\partial x}\left(\bar{\kappa} \frac{\partial T(H)}{\partial x}\right)
$$

The use of the enthalpy form for the energy conservation equation (equation 11) instead of the temperature form (equation 6) makes the problem easier to solve (appendix A).

\section{Application to the Congo continental slope}

One reason for the selection of the Congo continental slope as an application site for our model (Figure 3-a) was the observation of a sharp boundary between an upper slope area with a low seafloor reflectivity, as inferred from 3D seismic data (data available from TOTAL), and a lower slope area with a high seafloor reflectivity. The boundary was found to lie at a water depth that varied between two surveys performed at a 6 month time interval, from $545 \mathrm{~m}$ to $575 \mathrm{~m}$ (Figure 3). The 3D seismic data were collected using an airgun source. Received signals were sampled at $4 \mathrm{~ms}$. Used frequencies were in the range 3-100 Hz. Inline and cross line spacing were $25 \mathrm{~m}$.

Our working hypothesis was that the contrast in seafloor reflectivity could be related to a moving gas or gas hydrate front, at the upper limit of the gas hydrate stability zone, on the Congo continental slope. Two seismic sections (from the 3D seismic data) are presented in Figure 4, which show high reflectivity reflectors underlying the pockmark fields observed on the 3D seismic image of the seafloor (Figure 3-b). On the other hand, the limit between high and low reflectivity observed from the 3D seismic image (Figure 3-b) matches well with a lower reflection amplitude observed over a depth interval of around $30 \mathrm{~m}$ below seafloor for the section CS1 (Figure 4-a) and around $25 \mathrm{~m}$ for the section CS2 (Figure 4-b).

In addition to the 3D seismic data, various geophysical data were collected in the area during the Ifremer-Total ZaïAngo surveys in 1998, 1999 and 2000. These data include deep towed 
high-resolution sub-bottom profiles and side-scan sonar images. Side scan sonar images showed the existence of seafloor fissures and depressions (pockmarks) (Figure 5). The $3.5 \mathrm{kHz}$ profiles revealed high reflection amplitudes at shallow depth in the pockmark field. Whenever the plums reach the seafloor, pockmarks are observed (Figure 6). The high reflectivity could be related to carbonate crust occurrences.

\section{III.1 Thermal data on the Congo continental slope}

A first set of geothermal data was acquired on the Congo slope in December 1998. Location of these measurements as well as core stations acquired during several surveys in the studied area since 1998 are shown in Figure 5. The variation with time of the temperature at the seafloor interface was monitored during 8 months at two positions (moorings A7 and B7 Figure 5). At sites KZR_26, KZR_27 and FZ2_07, in-situ temperature measurements in the upper meters of sediment were made by means of thermistor probes attached to a $14 \mathrm{~m}$ long gravity corer. In Figure 7 are presented the temperature profiles with depth for the cores KZR_26, KZR_27 and FZ2_07. Excluding the uppermost temperature for each profile, mean geothermal gradients are obtained by least square regression (Figure 7). They are in a narrow range of values, from $48^{\circ} \mathrm{C} / \mathrm{km}$ to $56^{\circ} \mathrm{C} / \mathrm{km}$ (Table 2). Heat flow was calculated for each station as the simple product of the geothermal gradient and the mean thermal conductivity measured by the hot wire method on the recovered core (Table 2). The departure of the uppermost temperature on each profile from the mean linear trend suggests that a thermal disturbance probably caused by a recent variation of the bottom water temperature, has penetrated the seafloor down to a shallow depth.

III.1.1 Changes in the bottom water temperature inferred from temperature records at the mooring sites A7 and B7.

Long-term temperature measurements at mooring sites A7 and B7 (Figure 6) provide continuous temperature records at the seafloor for a period of 8 months, from May 2000 to 
December 2000. These data bear information on the temporal changes in the bottom water temperature as a function of depth on the Congo continental slope. Temperature records at the mooring sites $\mathrm{A} 7$ and $\mathrm{B} 7$ show temperature variations over various time scales (Figure 8), from fast changes over a few hours to slower changes over periods of several days or months. The temperature range at the shallower site A7 (480 m of water depth), from $6.0{ }^{\circ} \mathrm{C}$ to $9.0^{\circ} \mathrm{C}$, gets narrower at the deeper site $\mathrm{B} 7(730 \mathrm{~m})$, from $4.6^{\circ} \mathrm{C}$ to $5.7^{\circ} \mathrm{C}$. Temperature changes at the deeper site $\mathrm{B} 7$ tend to mimic the temperature changes at the shallower site $\mathrm{A} 7$, but with reduced amplitudes.

In order to determine periods of temperature variations, a discrete Fourier transform was applied to records at sites A7 and B7. For the A7 records, we have identify three main frequencies for temperature variation corresponding to: $\mathrm{f}_{1}=2.210^{-5} \mathrm{~Hz} ; \mathrm{f}_{2}=7.310^{-7} \mathrm{~Hz}$ and $f_{3}=210^{-7} \mathrm{~Hz}$. Those frequencies correspond respectively to the time-periods of 12.62 hours, 15.85 days and 57.87 days. Comparable frequencies $\left(\mathrm{f}_{1}=2.210^{-5} \mathrm{~Hz}\right.$ and $\mathrm{f}_{2}$ comprised between 7.5 $10^{-7} \mathrm{~Hz}$ and $8.510^{-7} \mathrm{~Hz}$ ) were determined for site B7. Thus, temperature variations observed at sites A7 and B7 can be analytically expressed by the following equation:

$$
T=T_{0}+a_{1} \sin \left(2 \pi f_{1} t+\varphi_{1}\right)+a_{2} \sin \left(2 \pi f_{2} t+\varphi_{2}\right)+a_{3} \sin \left(2 \pi f_{3} t+\varphi_{3}\right)
$$

where $t$ is the time, $T_{0}$ is an initial temperature, $a_{1}, a_{2}$ and $a_{3}$ are the amplitudes of the cyclic temperature variations and $\varphi_{1}, \varphi_{2}$ and $\varphi_{3}$ are the lags between the three cyclic temperature variations.

The parameters used in equation [12] can be identified by fitting the analytical expression to the observed data (Figure 8).

The temperature at the seafloor varies with periods of 12.6 hours, 13.6-16 days and about 59 days. The shorter periods correspond to tidal cycles but the 59 days periodicity is not understood. Indeed, the power spectral density of the tide cycles during the same period of the temperature measurements at site A7 and B7 shows two main periods of 12.87 hours and 
13.41 days which are similar to the temperature periods observed at the two sites A7 and B7 of 12.6 hours and 13.6-15.8 days. The temperature changes measured at different locations in the area at different water depths are reported in Figure 9. Thanks to the long-term temperature monitoring at sites A7 and B7 and to available data at greater depth from site MPL1 and MAP2 (Vangriesheim 2003), an upper bound and a lower bound of the temperature at the seafloor with increasing water depth are approximated by [13] and [14], with $\mathrm{z}$, the water depth, in metres.

$$
\begin{aligned}
& T_{\min }=4.0+(21.0-4.0) \exp (-0.0046 z) \\
& T_{\max }=4.0+(34.0-4.0) \exp (-0.0037 z)
\end{aligned}
$$

It will be noted that all bottom water temperatures recorded at the seafloor during the ZaïAngo geothermal measurements are positioned consistently in the temperature interval between these upper and lower bounds (Figure 9).

\section{III.2 Consequences of gas hydrate dynamics on the Congo continental slope.}

We examine in this section the geometry of the methane hydrate stability zone under two assumptions: 1) under steady state regime, by considering a constant seafloor temperature and 2) under transient regime, by considering the cyclic variation of the seafloor temperature presented in Figure 8, and applying the model proposed above.

\section{III.2.1 Steady state regime}

We use the methane hydrate stability law (equation [1]) and the methane (99\%)-ethane (1\%) hydrate stability curve (Sultan et al. 2002) to calculate the thickness of the gas hydrate stability zone (GHSZ) in the studied area. The input data consists of the seafloor bathymetry, the sea bottom temperature and the geothermal gradient in the sediments. Two extreme cases for the sea bottom temperature are considered: the low bound value (equation [13]) and the high bound one (equation [14]). From Figure 9, we used the intersection of the temperature 
profile with depth and the gas-hydrate stability curves in order to evaluate the thickness of the GHSZ. This varies from $525 \mathrm{~m}$ to $610 \mathrm{~m}$ (for $100 \%$ of methane) and from $480 \mathrm{~m}$ to $578 \mathrm{~m}$ (for $99 \%$ of methane and $1 \%$ of ethane). Figure 9 illustrates the importance of the chemical composition of the gas on the gas-hydrate stability law. Unfortunately, during the different ZaïAngo surveys, no geochemical data were acquired in the area. Our assumption of a mixed composition of methane and ethane is comforted by the geochemical data acquired in ODP hole 1077 (Berger et al. 1998 ). Hole 1077 is located around $35 \mathrm{~km}$ at the NE of the study area (Figure 3-a) where headspace analysis indicated the presence of biogenic methane with insignificant amounts of ethane (less than $10 \mathrm{ppm}$ ) and no discernable amounts of heavier hydrocarbons. We present in Figure 10-a and $\mathrm{b}$ a contour map defining the thickness of the GHSZ for two extreme values of the sea bottom temperature (equations 13 and 14) and assuming that the gas is pure methane.

From the 3D seismic data, the seafloor reflectivity contrast was found to lie at a water depth that varies with time, from $545 \mathrm{~m}$ for the first survey to $575 \mathrm{~m}$ for the second survey (Figure 3). It is worth noting at this stage that both of these values reflectivity contrast lie clearly between the extreme depths of $595 \mathrm{~m}$ and $610 \mathrm{~m}$ predicted for the gas hydrate stability front (Figure 9).

III.2.2 Transient regime: methane hydrate

In this paragraph, we simulate the dynamic of methane hydrate on the Congo continental slope, at a water depth of $557 \mathrm{~m}$. This depth corresponds to a median seafloor temperature of $6.3^{\circ} \mathrm{C}$ and to methane hydrate stability temperature of $6.37^{\circ} \mathrm{C}$ (Figure $11-\mathrm{a}$ and $\mathrm{b}$ ). The cyclic temperature variation (equation [12]) considered in the numerical simulation is presented in Figure 11-a. The parameters used in equation [12] are: 


$$
\left\{\begin{array}{l}
\mathrm{T}_{0}=6.3^{\circ} \mathrm{C} \\
\mathrm{f}_{1}=2.210^{-5} \mathrm{~Hz}, \mathrm{f}_{2}=7.310^{-7} \mathrm{~Hz}, \mathrm{f}_{3}=210^{-7} \mathrm{~Hz} \\
\mathrm{a}_{1}=0.2, \mathrm{a}_{2}=0.45, \mathrm{a}_{3}=0.4 \\
\varphi_{1}=0 ; \varphi_{2}=5.23598 ; \varphi_{3}=1.72785959
\end{array}\right.
$$

Other parameters used for the numerical simulation are given in Table 3. A sediment column of $20 \mathrm{~m}$ below the seabed is considered. The sediment column is divided into 2 layers. The porosity decreases linearly within each layer. The hydrate fraction is taken equal to $10 \%$ of the porosity from the seafloor to the base of the GHSZ. The initial condition corresponds to a steady state regime with the median sea bottom temperature $\left(6.3^{\circ} \mathrm{C}\right)$. The initial thickness of the MHSZ is equal to $2.2 \mathrm{~m}$ (Figure 11-b).

In Figure 12-a and $\mathrm{b}$ are presented (Figure 11-b) the resulting temperature and the hydrate field distribution within the sediments versus time for two periods (0-1000 days and $49000-$ 50000 days). From Figure 12-b, one can observe that the massif gas hydrate over the first $2.2 \mathrm{~m}$ will be reduced to about $0.5 \mathrm{~m}$. Indeed, because of the physical properties of the two phases of the gas hydrate the dissociation process is faster than the formation one. The thermal diffusivity $\left(=\frac{\kappa}{C}\right)$ of the hydrate phase $\left(2.1110^{-7} \mathrm{~m}^{2} / \mathrm{s}\right)$ is greater than the thermal diffusivity of the liquid phase $\left(1.4010^{-7} \mathrm{~m}^{2} / \mathrm{s}\right)$ and the thermal diffusivity of the gas phase. It is interesting to see that with the temperature fluctuations considered in the calculation it is possible to create theoretically, over the first 2 meters, two Bottom-Simulating Reflectors (BSR) corresponding to an acoustic impedance contrast between hydrate-bearing sediments and free gas trapped in the sediment (Figure 12-b). For temperature fluctuations with larger wavelength, these double BSRs can be observed at higher depth (see for instance Posewang and Mienert 1999 and Foucher et al. 2002). From the simulation results presented in Figure 12 , one can suggest that temperature fluctuations in the sediment wedge at the upper limit of the GHSZ on the Congo continental slope, as inferred from the limited time records of the sea 
bottom water temperature presented in the paper, tend to reduce the gas hydrate stability zone. This would be true in the sediment wedge wherever the pressure and temperature conditions for gas hydrate stability would not last permanently. In Figure 12, the exchange between the two phases gas and gas hydrate generated by the temperature fluctuations concerns only the first meter.

III.2.3 Transient regime: methane-ethane hydrate

In this paragraph, we consider the methane-ethane ( $99 \%$ and $1 \%$ respectively) as the free gas trapped in the sediment in order to identify the impact of the gas feeds on the gas hydrate stability zone on the Congo continental slope. The hydrate fraction is taken equal to $10 \%$ of the porosity from the seafloor to the base of the GHSZ. For this calculation, we consider the initial conditions of Figure 11-b, which corresponds to a steady state regime with a sea bottom temperature of $6.3^{\circ} \mathrm{C}$. For the stability law of the methane-ethane hydrate presented in Figure 9, the initial thickness of the GHSZ is equal to $17.2 \mathrm{~m}$. In Figure $13-\mathrm{a}$ and $\mathrm{b}$ are presented the resulting temperature and the hydrate field distribution within the sediments versus time for two periods (0-1000 days and $49000-50000$ days). Due to the high thermal diffusivity of the hydrate phase the depth of penetration of the temperature perturbations is around $6 \mathrm{~m}$. On the other hand, we did not observe any switch between the gas phase and the hydrate phase (Figure 13-a).

III.2.4 Migrating seafloor reflectivity and hydrate stability zone

The aim of the previous calculations (Figure 12 and Figure 13) was to verify the link between the reflectivity contrast on the Congo continental slope and its periodical displacement (Figure 3) and the possible migrating gas hydrate front. The different calculations results (Figure 12 and Figure 13) show that, for the temperature fluctuations presented in Figure 8, the switch between gas and hydrate phases occurs for methane hydrate and concerns only the first meter of sediment (Figure 12). Consequently, for the 6 months period separating the two 
seismic surveys (Figure 12-b: example between 49100 and 49300 days), over the first meter of sediment, a gas degree of saturation of around $2 \%$ (for calculation details see Sloan 1998) will replace the hydrate degree of saturation of around $8 \%$ (porosity*hydrate fraction). By using the effective medium theory for the calculation of the P-wave velocity for gassy soil and hydrate-bearing sediments (Helgerud et al. 1999), one can observe a change of the P-wave velocity from $1570 \mathrm{~m} / \mathrm{s}$ (for the $8 \%$ of hydrate) to around $1279 \mathrm{~m} / \mathrm{s}$ (for the $2 \%$ of gas saturation) over the first meter (Figure 14-a and -b). As it was mentioned before, the wavelength of the seismic signal is about $10 \mathrm{~m}$. Consequently, the seismic image of Figure 3$\mathrm{b}$ integrate the wave velocities and unit weights of the sediment over the first $10 \mathrm{~m}$. The two reflectance coefficient profiles determined for the two period times (6 months apart) over the first $10 \mathrm{~m}$ is presented in Figure 14-a and $-\mathrm{b}$. The tiny difference between the two maximum reflectance coefficients corresponding to the two time periods (survey 1 and survey 2) (in Figure 14) show that the shift between hydrate and gas over the first meter of sediment cannot induce an effect on the seismic image presented in Figure 3. In Figure 14-c, we have considered the case where the gas hydrates were completely replaced by gas over the first meter. The reflectance coefficients profile shown in Figure 14-c is similar to the two first reflectance profiles (Figure 14-a and -b). Consequently, in the conditions of our proposed model, our working hypothesis that the occurrence of the reflectivity contrast on the Congo continental slope and its periodical displacement (Figure 3) could be linked to a possibly migrating gas hydrate front appears to be ruled out. The question concerning the origin of the observed reflectivity contrast remains open. However, for temperature fluctuations with larger wavelength the switch between gas and gas hydrate will be deeper and consequently could explain the reflectivity contrast as a possible migrating gas or gas hydrate front. 


\section{Conclusion}

In this paper, a new numerical approach was developed to model the dynamic evolution of methane hydrate occurrences in marine sediments on a continental slope when the sea bottom temperature changes with time. This approach is based on the use of the enthalpy form of the law of conservation of energy, which made the problem numerically simple. Modelling the dynamics of gas hydrate sedimentary systems at the earth's surface is of importance to a variety of applications, including an assessment of the mechanisms through which large amounts of methane could be released to the atmosphere from dissociating gas hydrate in continental margins.

The response of gas hydrate-containing sediment to a time variation of the sea bottom temperature is not immediate. This is because of the slow process of heat propagation in the sediment that results from the low thermal diffusivity of the sediment but is also much influenced by the latent heat of gas hydrate formation or dissociation (Appendix B).

As an illustration of the capability of the modelling approach developed in this paper, we simulated dissociation of gas hydrate potentially present in sediments of the Congo continental slope in response to short-term sea bottom temperature fluctuations recorded over 8 months. An effect of the temperature fluctuations that the modelling approach illustrates well is the thermal erosion of shallow gas hydrate occurrences at the upper limit of the GHSZ on the continental slope. This may be viewed as an indication that gas hydrate would be stable only in the slope area defined from the least favourable temperature and pressure conditions for gas hydrate stability, in case those conditions fluctuate fast enough with time, such as observed on the Congo slope.

A primary objective of the present study was to check on whether gas hydrate dissociation could account for the seismic reflectivity contrast observed on the Congo continental slope. Short-term temperature fluctuations at the sea bottom as those reported in this paper are found 
to propagate too shallow into the sediment to generate the reflectivity contrast observed on the 3D seismic data. We would not however rule out as an explanation of this contrast a process of gas hydrate dissociation that would result from longer-term changes of the sea bottom temperature in the water depth interval on the Congo continental slope of importance to this study, between $500 \mathrm{~m}$ and $650 \mathrm{~m}$.

Acknowledgements. Authors are very grateful to B. Savoye head of the ZAIANGO project at IFREMER and A. Morash head of the Deep Offshore Project at TOTAL, for their financial support and data supplies. An insightful review of Dr. Pierre Henry, Prof. Jürgen Mienert and Prof. David Piper helped to improve an earlier version of the manuscript. 


\section{Appendix A}

Equation [11] can be written in a discrete form on a non-uniform grid by using a simple first order in time and second order in space. The discrete version of equation [11] can be written:

$$
\frac{H_{i}^{n+1}-H_{i}^{n}}{\Delta t}=-A_{i} T_{i-1}^{n+1}-B i T_{i}^{n+1}-C i T_{i+1}^{n+1}+D_{i}
$$

with:

$$
\begin{gathered}
A_{i}=-\frac{\alpha}{\Delta x_{i}} \frac{\bar{\kappa}_{i-1}}{x_{i}-x_{i-1}} \\
B_{i}=\frac{\alpha}{\Delta x_{i}}\left[\frac{\bar{\kappa}_{i}}{x_{i+1}-x_{i}}+\frac{\bar{\kappa}_{i-1}}{x_{i}-x_{i-1}}\right] \\
C_{i}=-\frac{\alpha}{\Delta x_{i}} \frac{\bar{\kappa}_{i}}{x_{i+1}-x_{i}} \\
D_{i}=\frac{1-\alpha}{\Delta x_{i}}\left[\bar{\kappa}_{i} \frac{\left.T_{i+1}^{n}-T_{i}^{n}-\bar{\kappa}_{i-1} \frac{T_{i}^{n}-T_{i-1}^{n}}{x_{i+1}-x_{i-1}}\right]}{x_{i}}\right]
\end{gathered}
$$

where $\mathrm{i}$ is the grid index, $\mathrm{n}$ is the time step index, $\Delta \mathrm{t}$ is the time step, and $\Delta \mathrm{x}$ is the grid spacing. The $\alpha$ value defines the type of the numerical method:

$$
\begin{cases}\alpha=0 & \text { explicit } \\ \alpha=1 & \text { fully implicit } \\ \alpha=0.5 & \text { Crank-Nicolson }\end{cases}
$$

This problem can be solved with a standard Newton method where the Jacobian is formed. Newton's method requires the solution of the linear system:

$$
\begin{aligned}
& J^{k} \delta H^{k}=-F\left(H^{k}\right) \\
& H^{k+1}=H^{k}+\delta H^{k}
\end{aligned}
$$


where $J$ is the jacobian matrix;

$F(H)$ is the nonlinear system of equations given by:

$$
F_{i}=\frac{H_{i}^{n+1}-H_{i}^{n}}{\Delta t}+A_{i} T_{i-1}^{n+1}+B_{i} T_{i}^{n+1}+C_{i} T_{i+1}^{n+1}-D_{i}
$$

$H$ is the enthalpy state vector;

And $k$ is the nonlinear iteration index.

For a one-dimensional problem, equation [A-7] is discretized into $\mathrm{n}$ equations and $\mathrm{n}$ unknowns where:

$$
F(H)=\left\{F_{1}, F_{2}, \ldots \ldots, F_{i}, \ldots \ldots, F_{N}\right\}
$$

and

$$
H=\left\{H_{1}, H_{2}, \ldots \ldots, H_{i}, \ldots \ldots, H_{N}\right\}
$$

Where $\mathrm{i}$ is the one-dimensional grid index. In vector notation, the $(i, j)^{\text {th }}$ element of the Jacobian matrix is given by:

$$
J_{i, j}=\frac{\partial F_{i}(H)}{\partial H_{j}}
$$




\section{Appendix B}

In order to identify the role of the latent heat on the heat transfer within a porous medium containing hydrate, the initial temperature profile of Figure 11-b was again considered but without latent heat. Simulation results are presented in Figure-B 1. Comparison between Figure 12-b and Figure-B 1-b, show clearly that the latent heat controls the results. When the latent heat is included (Figure 12-a), the time that it takes for the hydrate front to reach a given point is larger. These results were expected since the latent heat of the hydrate represents an additional heat sink for the energy equation (Briaud and Chaouch 1997). The comparison between the temperature contours shows once again the importance of the latent heat on the heat transfer within a porous medium holding hydrate. However, it is important to notice that the effect of the latent heat on the heat transfer depends strongly on the hydrate fraction. 


\section{REFERENCES.}

Berger, W., Wefer, G., Richter, C., et al., 1999. Proc. ODP, Init. Repts., 175: College Station, TX (Ocean Drilling Program).

Briaud, J.L., Chaouch, A., 1997. Hydrate melting in soil around hot conductor. Journal of Geotechnical and Geoenvironmental Engineering 123 (7), 645-653.

Chaouch, A., Briaud, J.-L., 1997. Post melting behavior of gas hydrates in soft ocean sediments. Proc. Offshore Technology Conference 8298, pp. 217-224.

Davie, M. K., Buffett, B. A., 2001. A numerical model for the formation of gas hydrate below the seafloor. J. Geophys. Res. 106 (B1), 497-514.

Delisle, G., Beiersdorf, H., Neben, S., Steinmann, D., 1998. The geothermal field of the North Sulawesi accretionary wedge and a model on BSR migration in unstable depositional environments. in Henriet, J.-P.; Mienert, J. (Ed.): Gas hydrates: relevance to world margin stability and climate change. Geological Society Special Publication, 137. The Geological Society: London, UK, pp. 267-274.

Dickens, G.R., Quinby-Hunt, M.S., 1994. Methane hydrate stability in seawater. Geophysical Research Letters 21 (19), 2115-2118.

Egeberg, P.K., Dickens, G.R., 1999. Thermodynamic and pore water halogen constraints on gas hydrate distribution at ODP Site 997 (Blake Ridge). Chem. Geol. 153, 5379 .

Foucher, J.P., Nouzé, H., Henry, P., 2002. Observation and tentative interpretation of a double BSR on the Nankai slope. Marine Geology 187, 161-175.

Furbish, D. J., 1997. Fluid physics in Geology. Oxford University press.

Grevemeyer, I., Villinger H., 2001. Gas hydrate stability and the assessment of heat flow through continental margins. Geophysical Journal International 145, 647-660.

Handa, Y.P., Stupin, D., 1992. Thermodynamic properties and dissociation characteristics of methane and propane hydrates in $70-\AA$-radius silica-gel pores. J. Phys. Chem. 96, 8599-8603.

Helgerud, M. B., Dvorkin, J., Nur., A., Sakai, A., Collett, T., 1999. Elastic-wave velocity in marine sediments: effective medium modelling. Geophys. Res.Lett. 26 (13), 20212024.

Henry, P., Thomas, M., Clennell, M.B., 1999. Formation of Natural Gas Hydrates in Marine Sediments 2. Thermodynamic Calculations of Stability Conditions in Porous Sediments. J. Geophys. Res. 104, 23005-23022.

Mienert, J., Posewang J., Lukas, D., 2001. Changes in the Hydrate Stability Zone on the Norwegian Margin and their Consequence for Methane and Carbon Releases into the Oceanosphere. In: Schäfer, P., Ritzrau, W., Schlüter, M., and Thiede (Eds.), The Northern North Atlantic: A changing Environment, Springer Verlag, New York, pp. 281-290. 
Patek, J., Klomfar, J., 2002. Measurement of the thermal conductivity of argon and methane: a test of a transient hot-wire apparatus. Fluid Phase Equilibria 198(1), 147-163.

Posewang, J., Mienert, J., 1999. The enigma of double BSRs: Indicators for changes in the hydrate stability field. Geo-Marine Letters 19, 157-163.

Rempel, A.W., Buffett, B.A., 1997. Formation and accumulation of gas hydrate in porous media. Journal of Geophysical Research 102 (B5), 10151-10164.

Rempel, A.W. and Buffett, B.A., 1998. Mathematical models of gas hydrate accumulations. in Henriet, J.-P. and Mienert, J. (eds); Gas hydrates: Relevance to world margin stability and climatic change, Geological Society of London, Special Publication, 137, pp. 63-74.

Sloan, E.D. Jr., 1998. Clathrate hydrates of natural gases. Marcel Dekker Inc., 2nd edition, New York.

Sultan N., Cochonat P., Foucher J.P., Cauquil E., 2002. Study of the affects of gas hydrates on the seafloor instability in the lower Congo basin: a thermodynamic chemical approach. Proc. Offshore site investgation and geotechnics " diversity and sustainability ". London, pp. 55-73.

Vagner, P. 2003. Comparaison des imageries acoustiques ZaïAngo. Report DIT.DRO/GM 2003 - 27, Ifremer, France.

Vangreishiem, A., 2003. Personal communication.

$\mathrm{Xu}, \mathrm{W} .$, Ruppel, C., 1999. Predicting the occurrence, distribution and evolution of methane gas hydrates in porous marine sediment. Journal of Geophysical Research 104, 50815096. 


\begin{tabular}{lcc}
\hline PARAMETERS & SYMBOL & UNITS \\
\hline Number of Layers & $\mathrm{n}$ & {$[-]$} \\
Layers depth & $z_{i}$ & {$[\mathrm{~m}]$} \\
Porosity (linear for each layer) & $\phi_{i}$ & {$[-]$} \\
Hydrate Fraction (linear for each layer) & $\eta_{i}$ & {$[-]$} \\
Unit weight (for each phase) & $\rho_{l}, \rho_{s}, \rho_{h}$ & {$\left[\mathrm{kN} \mathrm{m}^{-3}\right]$} \\
Thermal Conductivity (for each phase) & $\kappa_{l}, \kappa_{s}, \kappa_{h}, \kappa_{g}$ & {$\left[\mathrm{w} \mathrm{K}^{-1} \mathrm{~m}^{-1}\right]$} \\
Specific Heat (for each phase) & $C_{l}, C_{s}, C_{h}$ & {$\left[\mathrm{~J} \mathrm{~K}^{-1} \mathrm{~m}^{-3}\right]$} \\
Bathymetry & $d$ & {$\left[\mathrm{~m}^{-3}\right.$} \\
Stability law of gas-hydrate & $T_{m}(p)$ & {$\left[\mathrm{K}^{2}\right.$} \\
Latent Heat & $L$ & {$\left[\mathrm{~J} \mathrm{~m}^{-3}\right]$} \\
Time Increment & $\alpha t$ & {$[\mathrm{~s}]$} \\
Number of Time Increments & $\alpha t$ & {$[-]$} \\
Numerical Parameter & & {$[-]$} \\
\hline
\end{tabular}

Table 1. Model parameters. 


\begin{tabular}{cccccc}
\hline CORE & DATE & $\begin{array}{c}\text { WATER } \\
\text { DEPTH } \\
(\mathbf{m})\end{array}$ & $\begin{array}{c}\text { BOTTOM } \\
\text { WATER } \\
\text { TEMPERATURE } \\
\left({ }^{\circ} \mathbf{C}\right)\end{array}$ & $\begin{array}{c}\text { THERMAL } \\
\text { GRADIENT } \\
\left({ }^{\circ} \mathbf{C} / \mathbf{k m}\right)\end{array}$ & $\begin{array}{c}\text { FLUX } \\
\left(\mathbf{m} \mathbf{W m}^{-2}\right)\end{array}$ \\
\hline KZR_26 & $22 / 12 / 2000$ & 502 & 7.190 & 50 & 47.7 \\
KZR_27 & $22 / 12 / 2000$ & 550 & 6.850 & 56 & 61.5 \\
FZ2_07 & $19 / 11 / 1998$ & 557 & 6.628 & 48 & 37.3 \\
A7 & $1 / 05 / 2000 \rightarrow$ & 480 & $6.0<\mathrm{T}<9.0$ & - & - \\
& $23 / 12 / 2000$ & & & & - \\
B7 & $1 / 05 / 2000 \rightarrow$ & 730 & $4.6<\mathrm{T}<5.7$ & & \\
& $23 / 12 / 2000$ & & & & \\
\hline
\end{tabular}

Table 2. Thermal data from the Congo slope. 
PARAMETERS

SYMBOLS and UNITS

VALUES

\begin{tabular}{lcc}
\hline Number of Layers & $\mathrm{n}[-]$ & 2 \\
\hline Layers depth & $z_{i}[\mathrm{~m}]$ & 10 \\
& & 20 \\
\hline Porosity (linear for each layer) & $\phi_{i}[-]$ & $0.846-0.740$ \\
& & $0.740-0.726$ \\
\hline Hydrate Fraction (linear for each layer) & $\eta_{i}[-]$ & 0.1 \\
Unit Weight (for each phase) & $\rho_{l}, \rho_{s}, \rho_{h}\left[\mathrm{kN} \mathrm{m}^{-3}\right]$ & $10,26,9.2$ \\
Thermal Conductivity (for each phase) & $\kappa_{l}, \kappa_{s}, \kappa_{h}, \kappa_{g}\left[\mathrm{w} \mathrm{K}^{-1} \mathrm{~m}^{-1}\right]$ & $0.6^{*}, 2.5^{*}, 0.4^{*}$, \\
& & $0.037^{* *}$
\end{tabular}

Specific Heat (for each phase)

$C_{l}, C_{s}, C_{h}\left[\mathrm{~J} \mathrm{~K}^{-1} \mathrm{~m}^{-3}\right]$

$4.18710^{6}, 2.510^{6}$

$1.910^{6 * * *}$

\begin{tabular}{lll}
\hline Bathymetry & $d[\mathrm{~m}]$ & 557
\end{tabular}

Stability law of methane-hydrate

$T_{m}(p)[\mathrm{K}]$

$9.4946 \ln \left(\mathrm{p} / \mathrm{p}_{0}\right)+263.212$ where $p$ is the hydrostatic pressure in $\mathrm{MPa}$ and $\mathrm{p}_{0}$ is a reference pressure

Latent Heat

$L\left[\mathrm{~J} \mathrm{~m}^{-3}\right] \quad 410^{8 * * *}$

Time Increment

$\Delta t[\mathrm{~s}]$

8640

Numerical Parameter

$\alpha[-]$

1

* from Grevemeyer and Villinger 2001

** from Patek and Klomfar 2002

*** from Briaud et Chaouch 1997

Table 3. Parameters used for the simulation of the methane hydrate stability zone at $557 \mathrm{~m}$ depth on the Congo slope. 


\section{FIGURES}

Figure 1. Methane hydrates stability law based on experimental results from Dickens and Quinby-Hunt (1994).

Figure 2. Temperature-enthalpy diagram

Figure 3. a) Bathymetry map showing the studied area, the ODP leg 175 drill sites and the locations of cores and heat flow measurements b) Seafloor reflectivity image showing a shift by $30 \mathrm{~m}$ (from $545 \mathrm{~m}$ to $575 \mathrm{~m}$ ) of the water depth at which the backscatter energy changes from high (dark) to low (light) between survey 1 and survey 2.

Figure 4. Cross sections across the limit between high and low backscatter a) CS1 and b) CS2 (refer to Figure 3 for the location of CS1 and CS2).

Figure 5. Side scan sonar (SAR) imagery (uncorrected for unbalanced antenna) with respect to a) locations of KZR_25, KZR_26, KZR_27, FZ2_07, A7 and B7 and b) to pockmarks and shallow faults.

Figure 6. $3.5 \mathrm{kHz}$ profile across the pockmark field of Figure 5.

Figure 7. Geothermal gradient from KZR_26, KZR_27 and FZ2_07.

Figure 8 . Bottom seawater temperature histories measured during 8 months compared to the analytical expression used in equation 12 a) site A7 (480 m water depth) and b) site B7 (730 m water depth).

Figure 9. Bottom seawater temperature (with observed range of variation in light grey) versus bathymetry - Congo slope. In the same diagram is presented the stability laws of methane hydrate and methane with ethane hydrate. 
Figure 10. Thickness of the methane-hydrate stability layer (steady state regime) a) for the minimum observed BWT profile and $\mathrm{b}$ ) for the maximum observed BWT profile (refer to Figure 3 for area location).

Figure 11. a) Analytical expression of the temperature change at a $557 \mathrm{~m}$ water depth and b) the initial temperature profile considered in the calculation.

Figure 12. a) Temperature and hydrate fields in the upper $10 \mathrm{~m}$ of the Congo slope, at a water depth of $557 \mathrm{~m}$, calculated for the initial temperature profile presented in Figure 11-b and for BWT fluctuations as shown in Figure 11-a; b) methane hydrate fields.

Figure 13. a) Temperature in the upper $10 \mathrm{~m}$ of the Congo slope, at a water depth of $557 \mathrm{~m}$, calculated for the initial temperature profile presented in Figure 11-b, for BWT fluctuations as shown in Figure 11-a and for a hydrate latent heat equal to zero b) methane-ethane $(99 \%$ and $1 \%$ respectively) hydrate fields.

Figure 14. P-wave velocity, unit weight and reflectance versus depth determined for a) hydrate - saturated soil interface, b) gas, hydrate and saturated soil interfaces and c) gassaturated soil interface.

Figure-B 1. a) Temperature in the upper $10 \mathrm{~m}$ of the Congo slope, at a water depth of $557 \mathrm{~m}$, calculated for an initial temperature profile presented in Figure 11-b, for BWT fluctuations as shown in Figure 11-a and for a hydrate latent heat equal to zero b) methane hydrate fields for zero latent heat. 


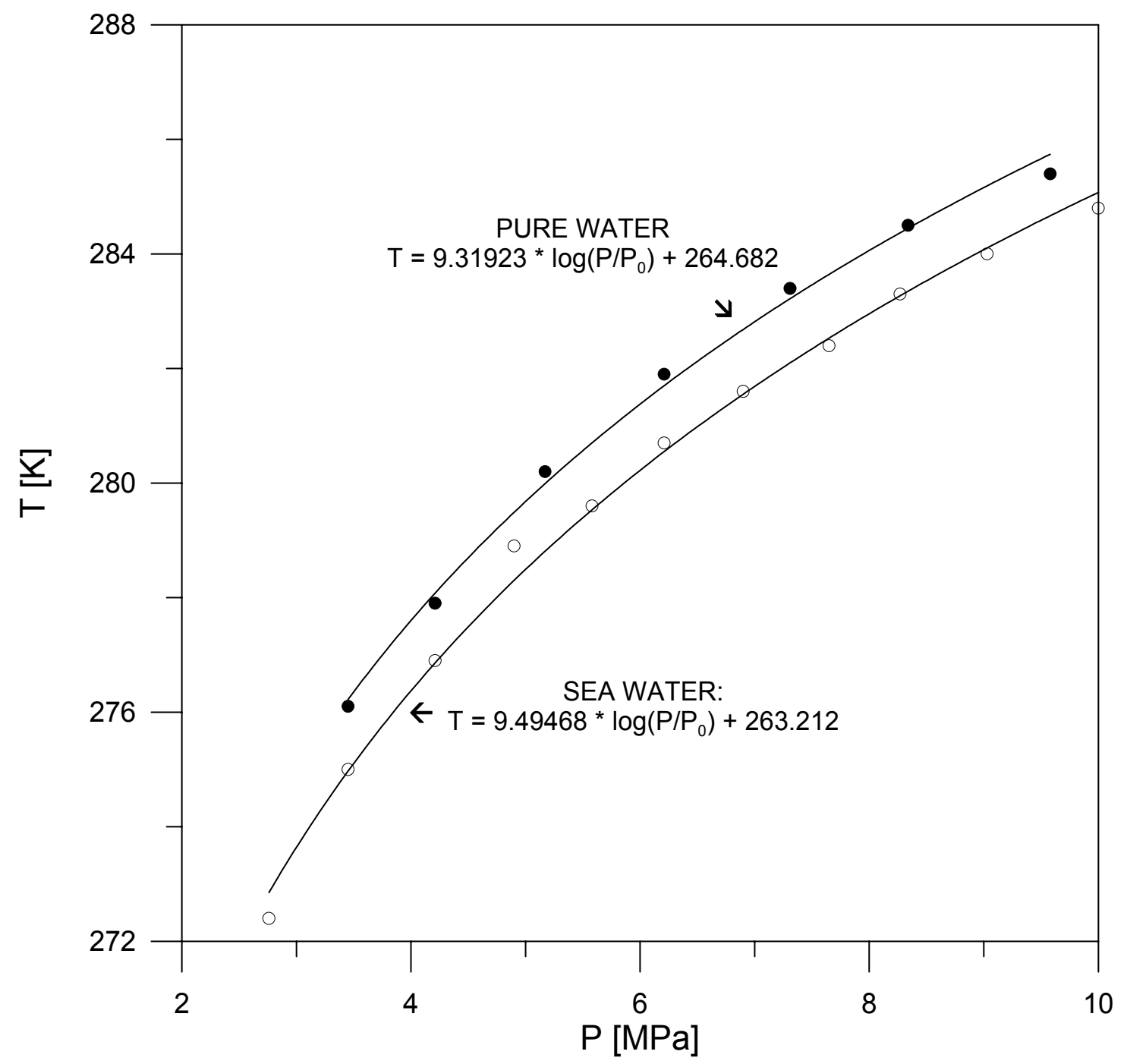

Figure 1. Methane hydrates stability law based on experimental results from Dickens and Quinby-Hunt (1994). 


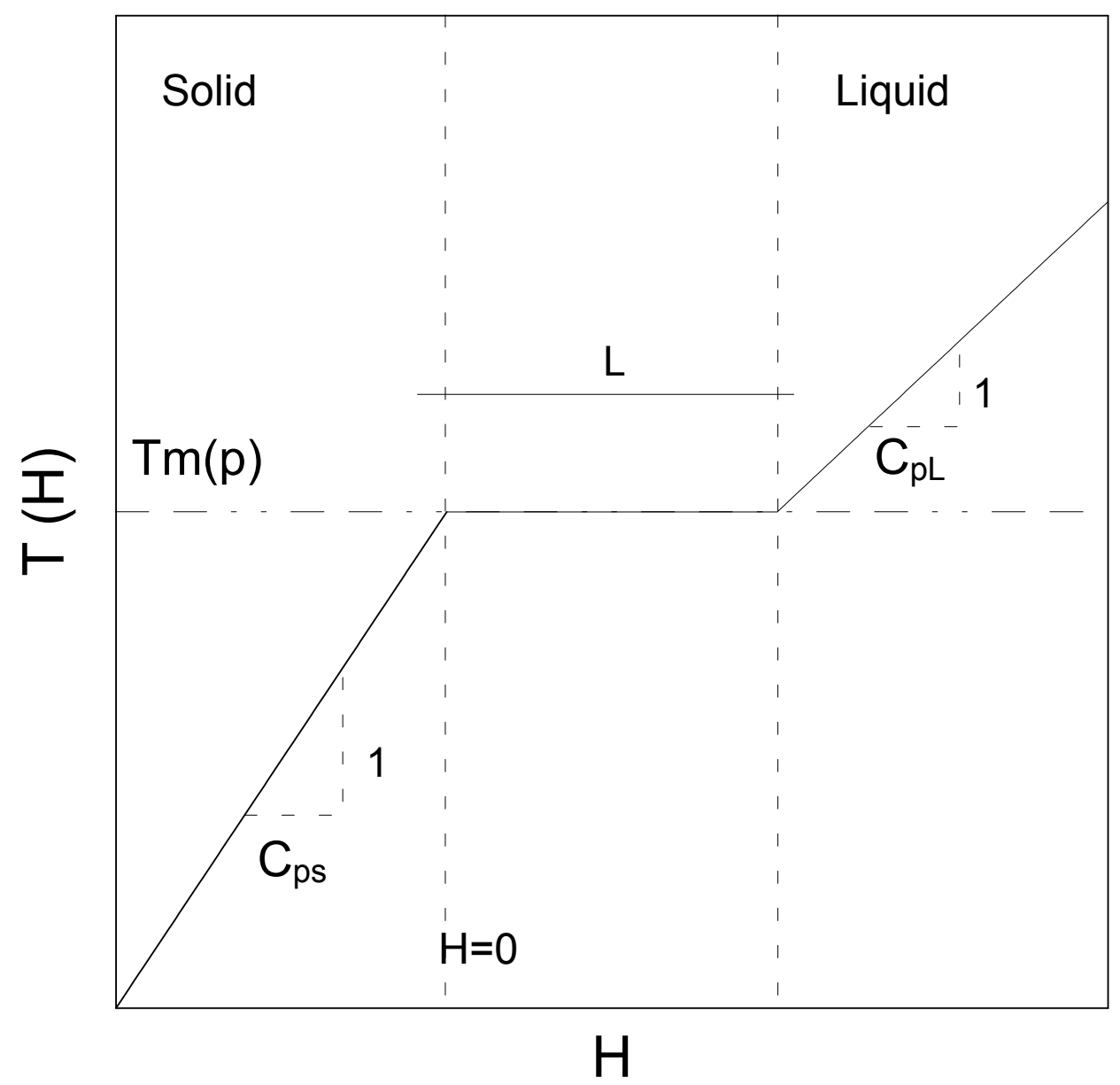

Figure 2. Temperature-enthalpy diagram 


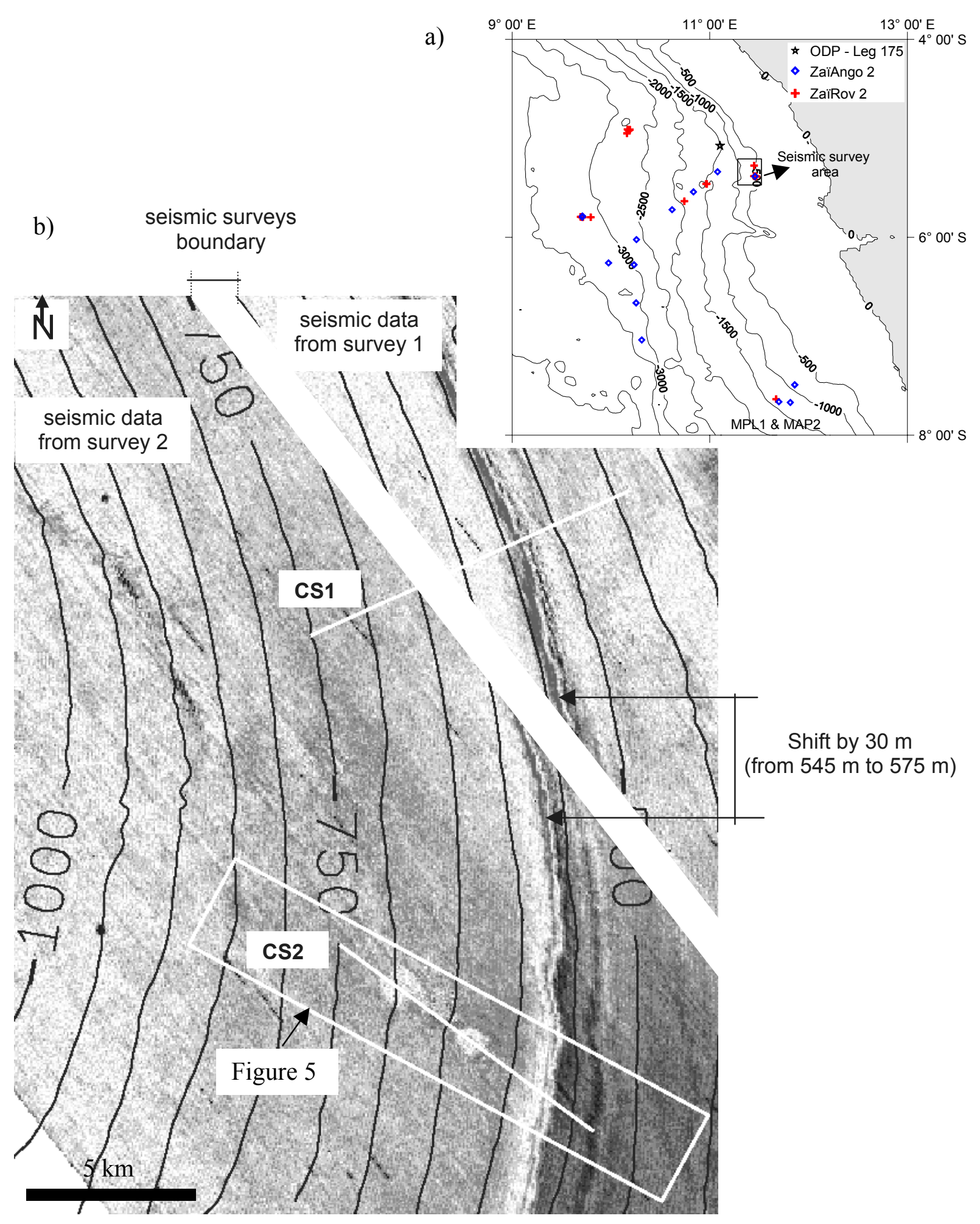

Figure 3. a) Bathymetry map showing the studied area, the ODP leg 175 drill sites and the locations of cores and heat flow measurements b) Seafloor reflectivity image showing a shift by $30 \mathrm{~m}$ (from $545 \mathrm{~m}$ to $575 \mathrm{~m}$ ) of the water depth at which the backscatter energy changes from high (dark) to low (light) between survey 1 and survey 2. 


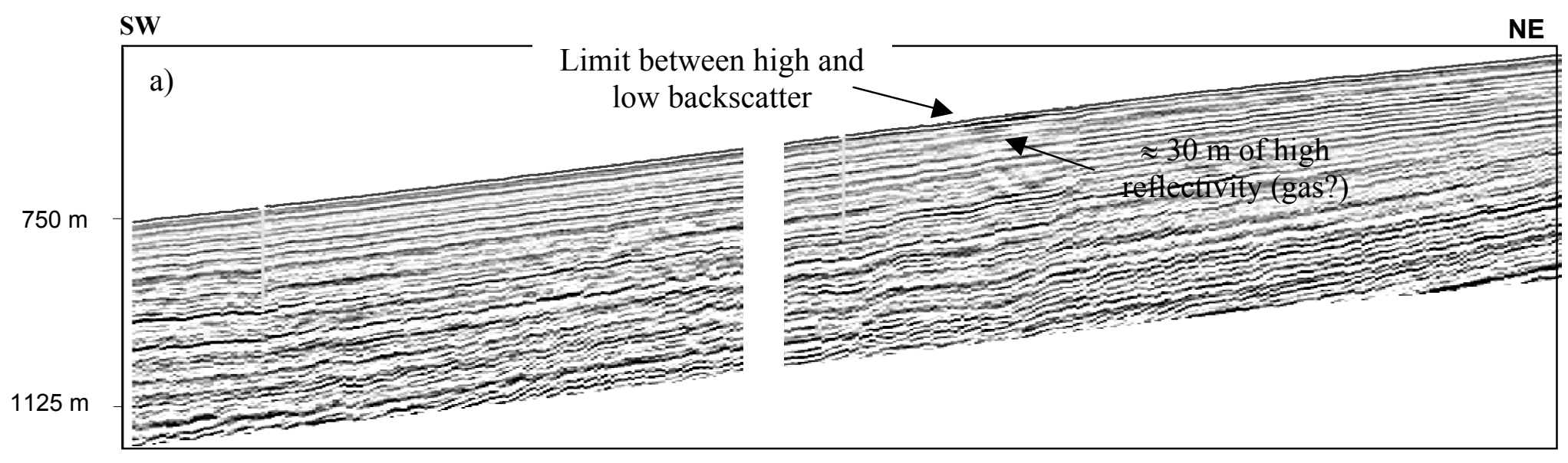

NE

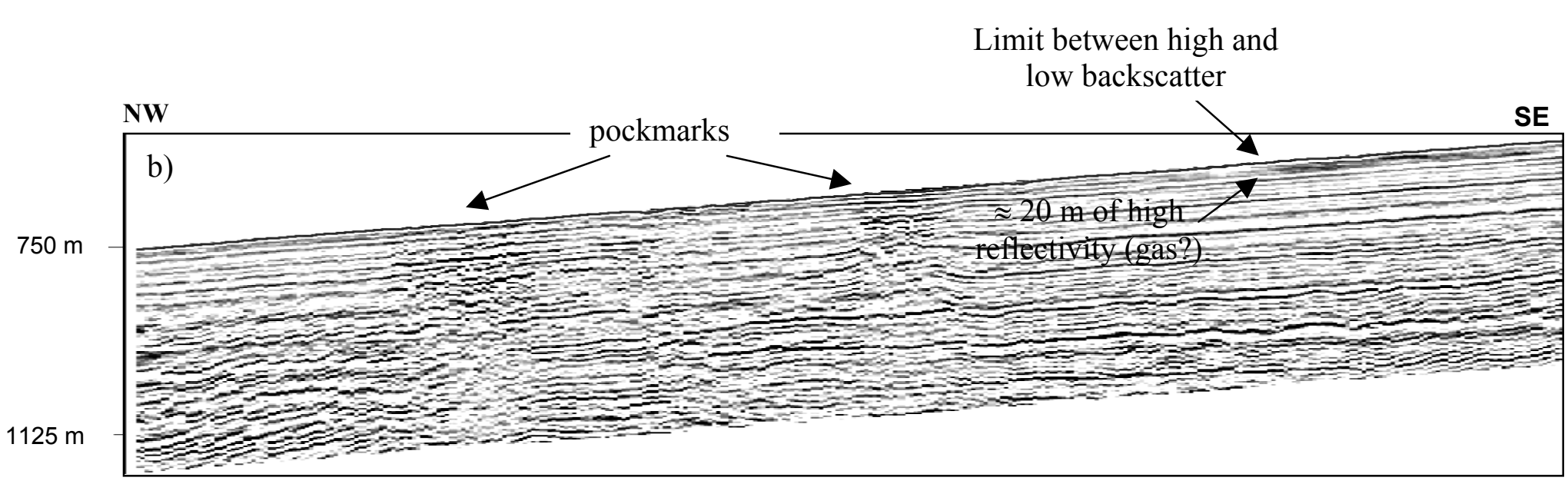

Figure 4. Cross sections across the limit between high and low backscatter a) CS1 and b) CS2 (refer to Figure 3 for the location of CS1 and CS2). 

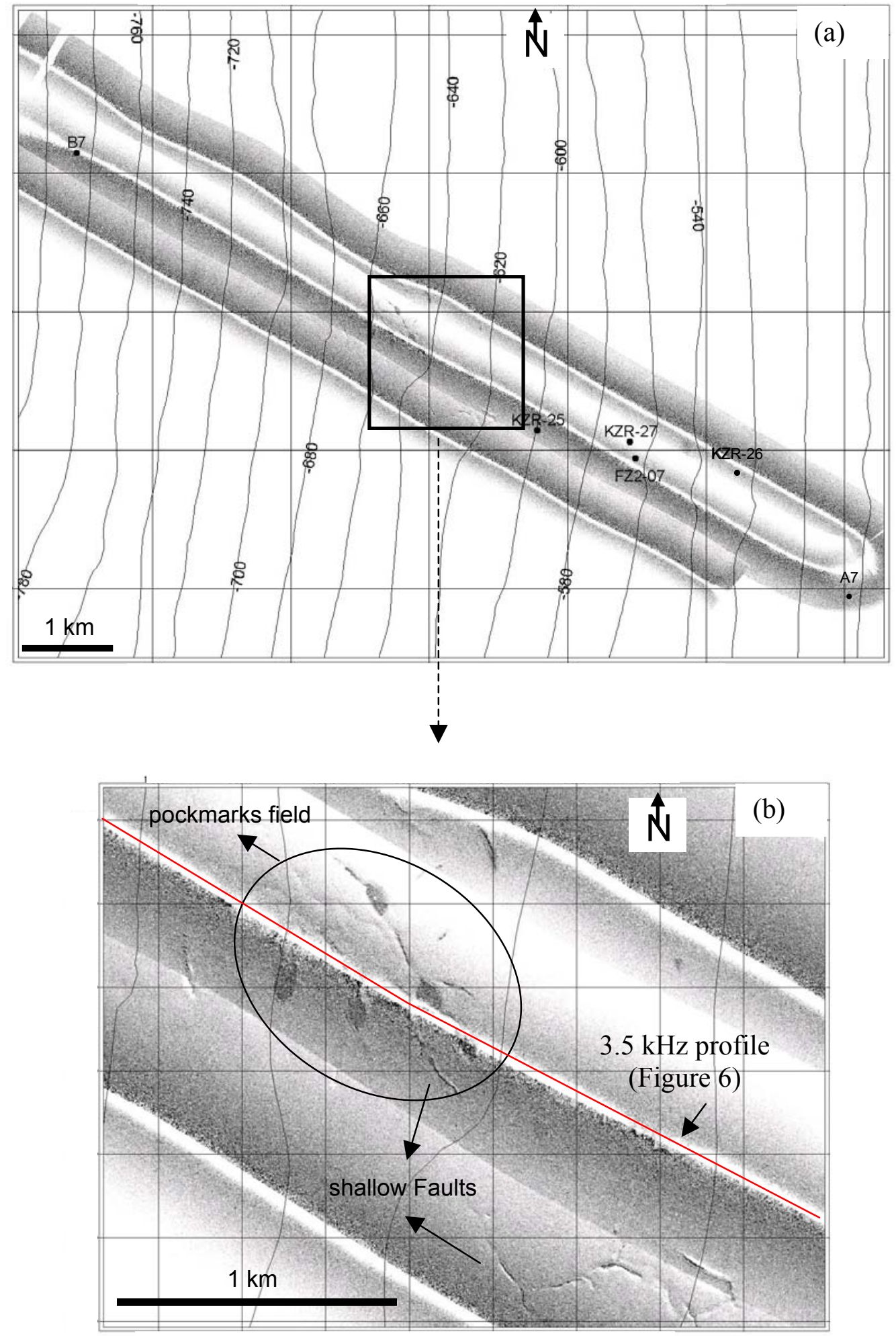

Figure 5. Side scan sonar (SAR) imagery (uncorrected for unbalanced antenna) with respect to a) locations of KZR_25, KZR_26, KZR_27, FZ2_07, A7 and B7 and b) to pockmarks and shallow faults. 


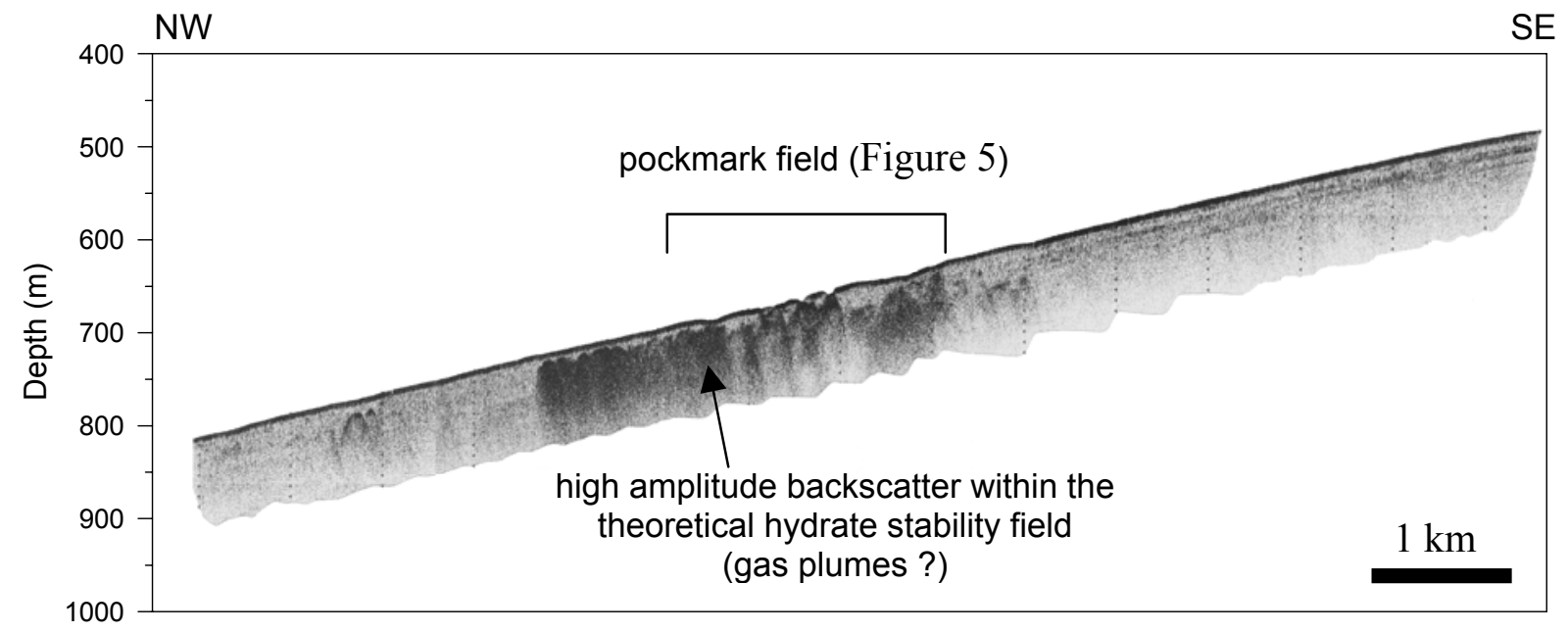

Figure 6. $3.5 \mathrm{kHz}$ profile across the pockmark field of Figure 5. 

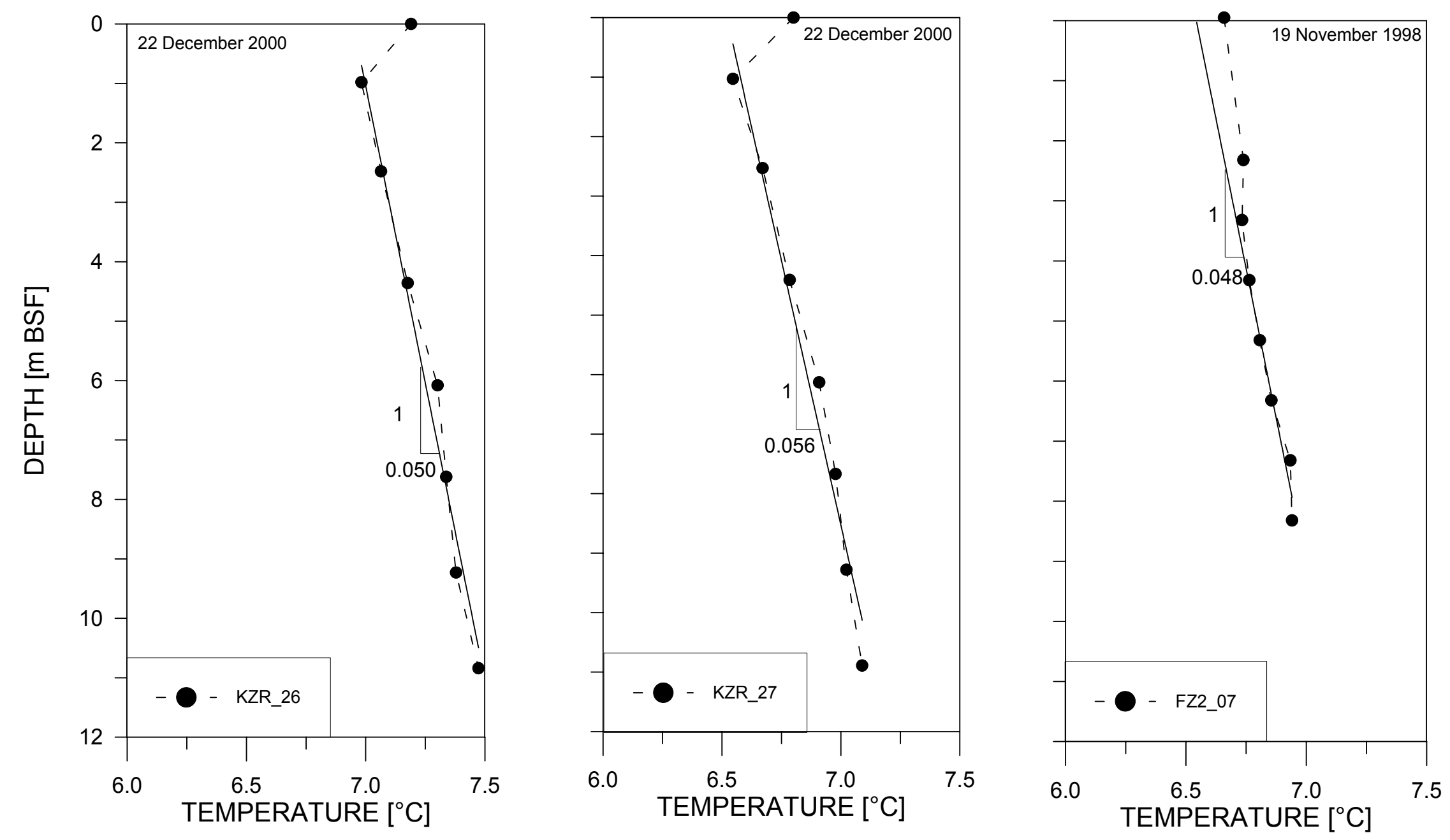

Figure 7. Geothermal gradient from KZR_26, KZR_27 and FZ2_07. 
Time (Hours)

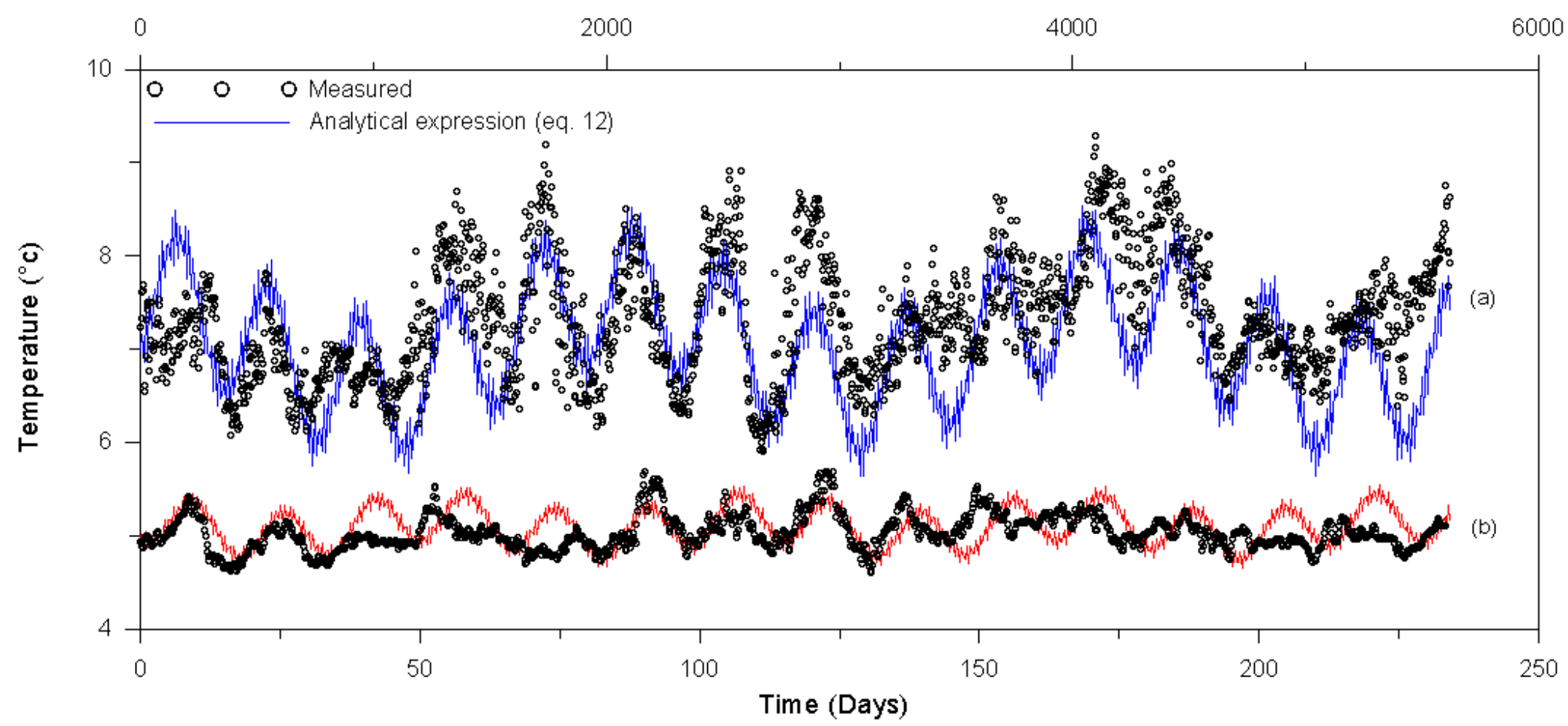

Figure 8. Bottom seawater temperature histories measured during 8 months compared to the analytical expression used in equation 12 a) site A7 (480 m water depth) and b) site B7 (730 m water depth). 


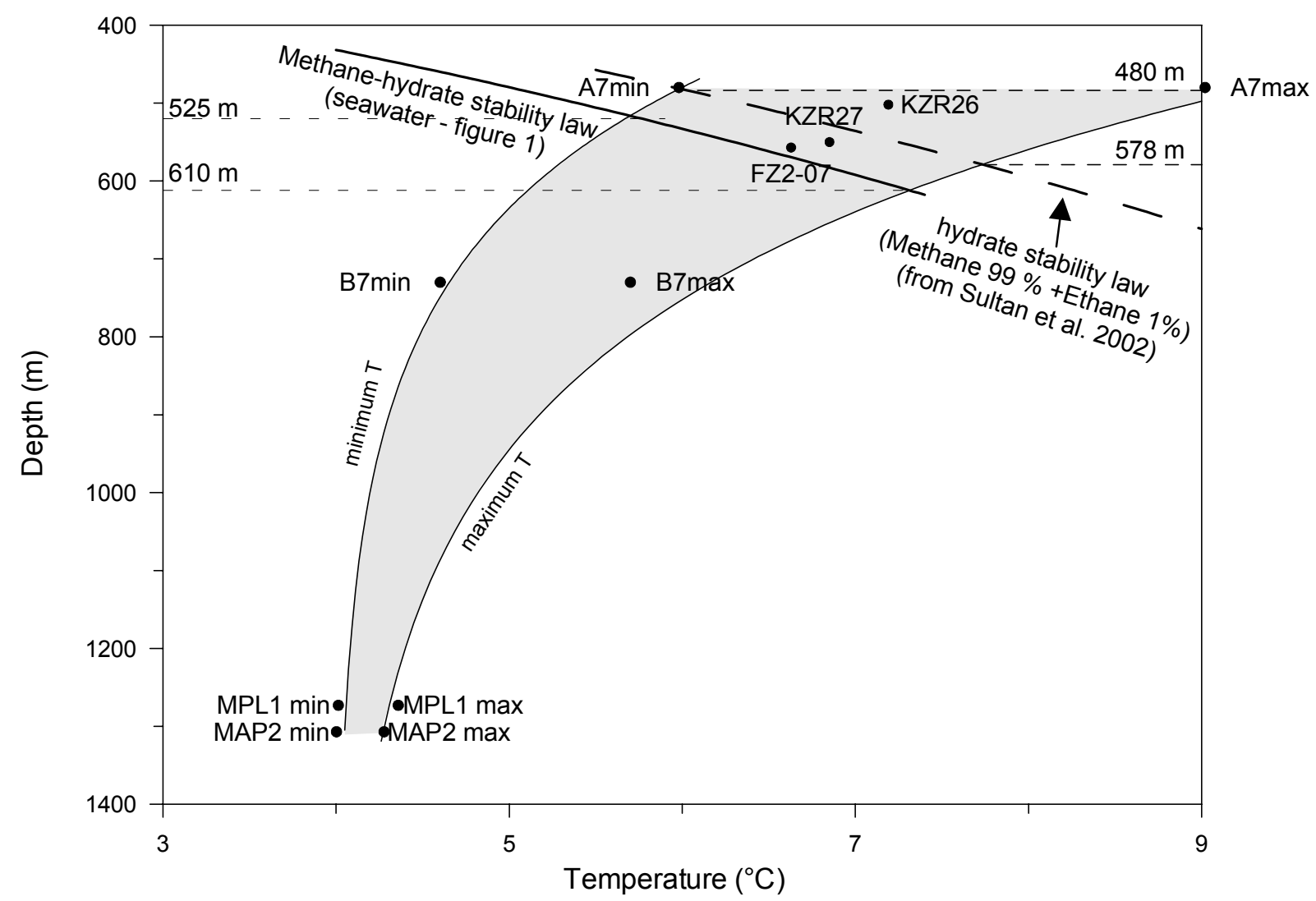

Figure 9. Bottom seawater temperature (with observed range of variation in light grey) versus bathymetry - Congo slope. In the same diagram is presented the stability laws of methane hydrate and methane with ethane hydrate. 

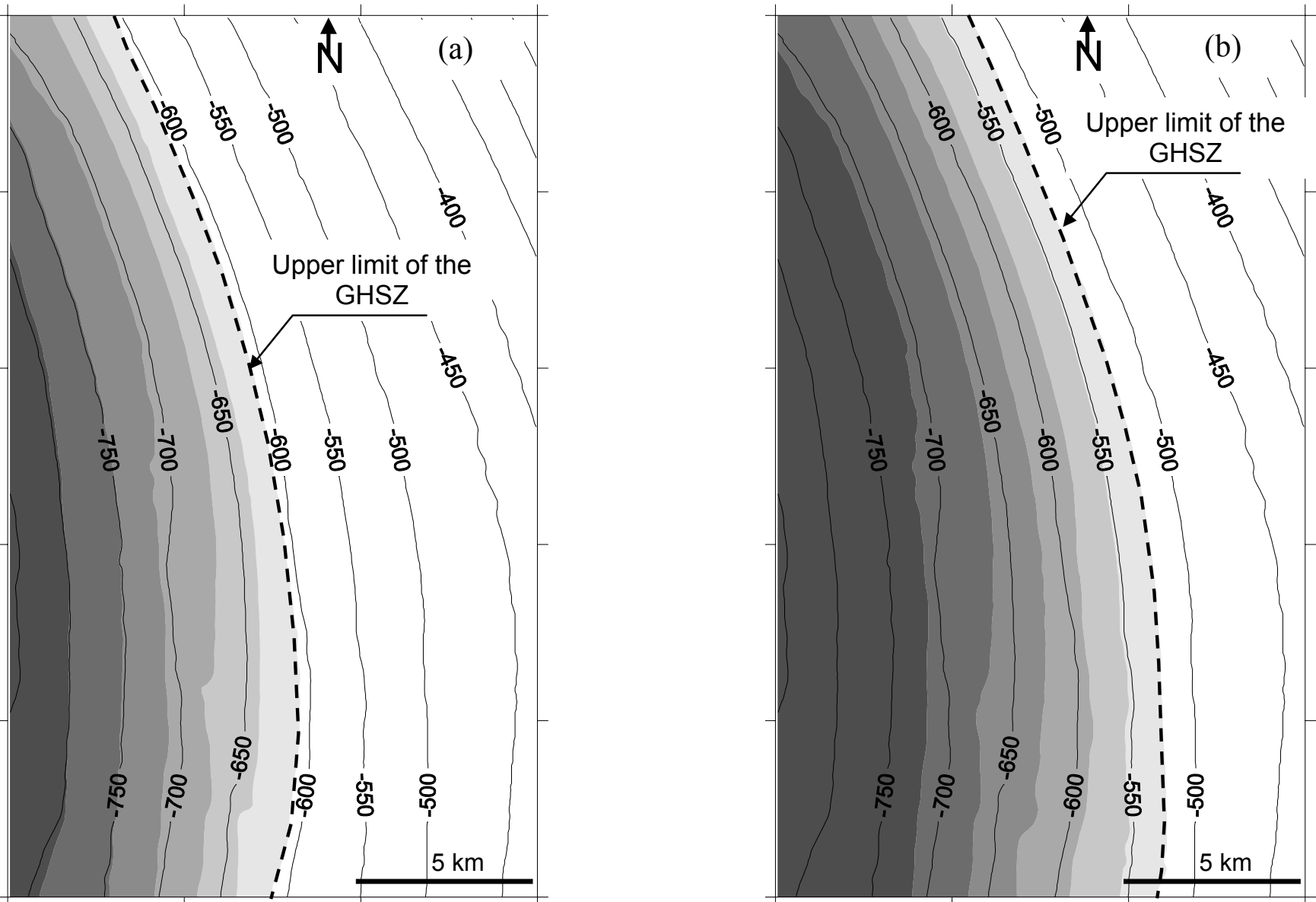

(m BSF)

Figure 10. Thickness of the methane-hydrate stability layer (steady state regime) a) for the minimum observed BWT profile and b) for the maximum observed BWT profile (refer to Figure 3 for area location). 

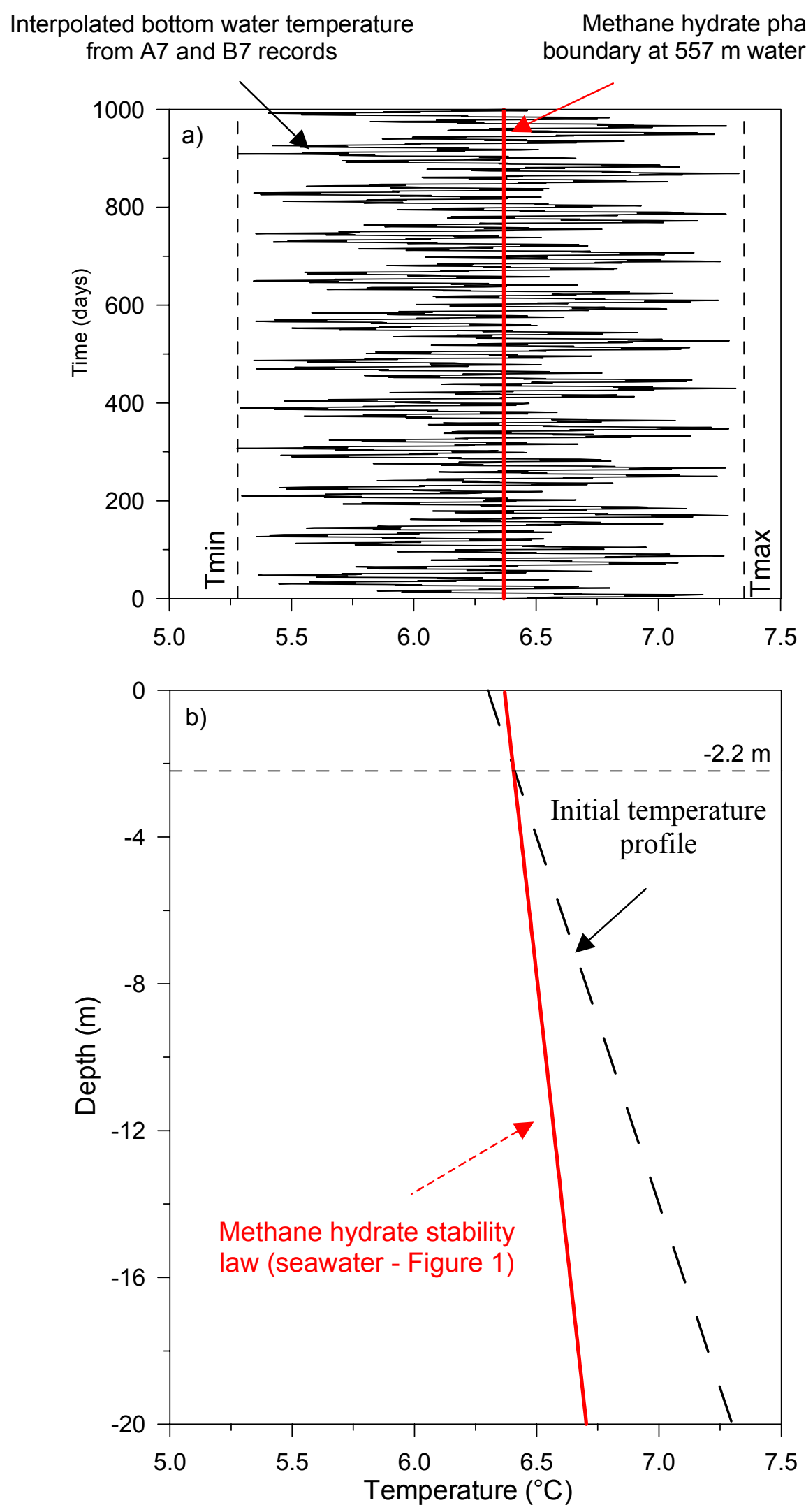

Figure 11. a) Analytical expression of the temperature change at a $557 \mathrm{~m}$ water depth and b) the initial temperature profile considered in the calculation. 

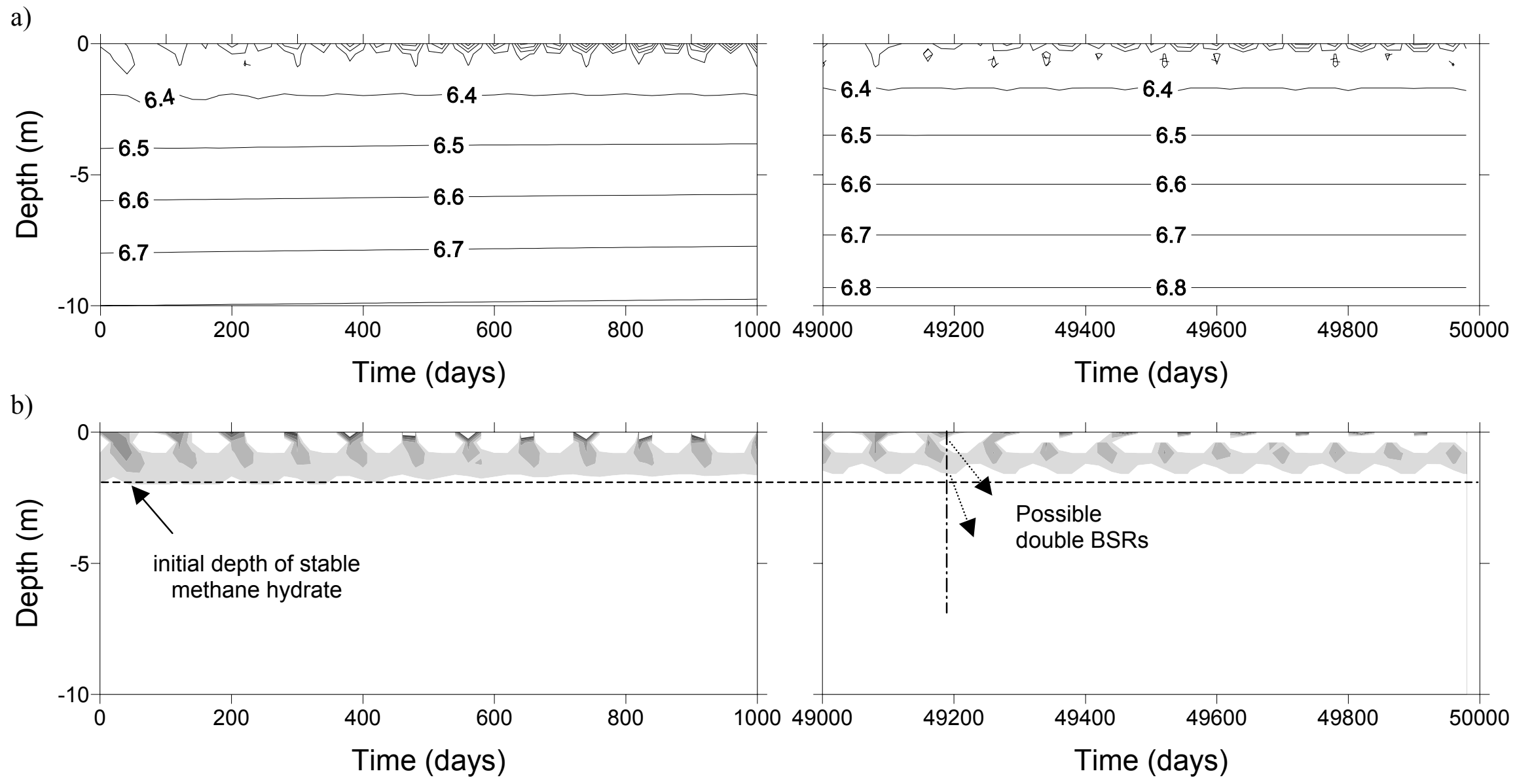

Figure 12. a) Temperature and hydrate fields in the upper $10 \mathrm{~m}$ of the Congo slope, at a water depth of $557 \mathrm{~m}$, calculated for the initial temperature profile presented in Figure 11-b and for BWT fluctuations as shown in Figure 11-a; b) methane hydrate fields 
a)

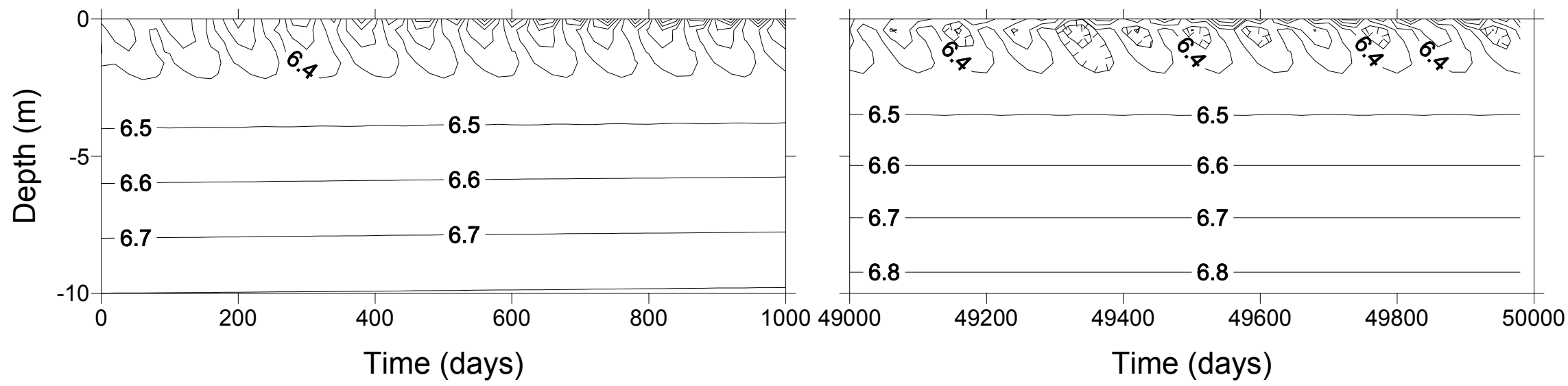

b)

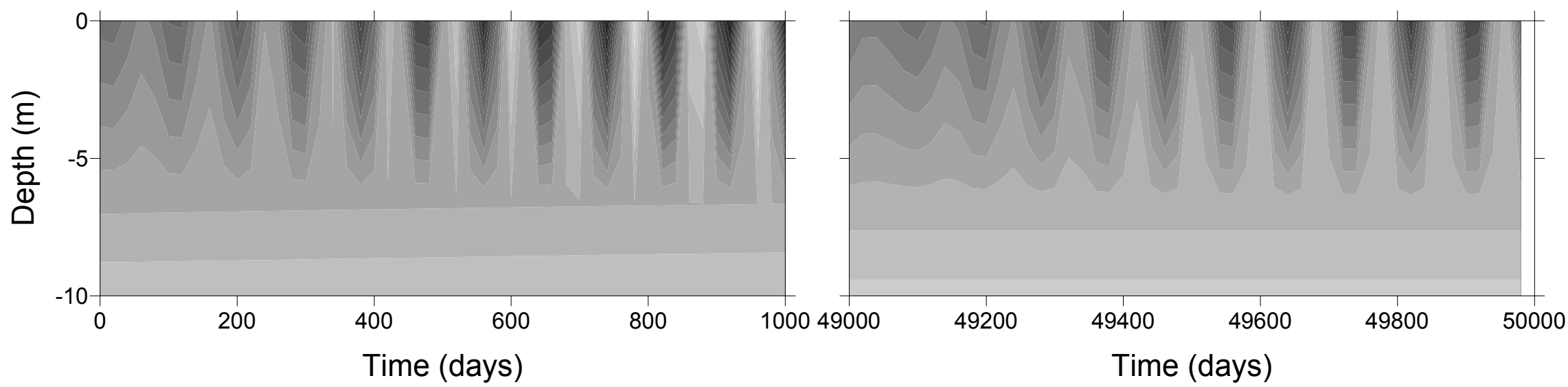

Figure 13. a) Temperature in the upper $10 \mathrm{~m}$ of the Congo slope, at a water depth of $557 \mathrm{~m}$, calculated for the initial temperature profile presented in Figure 11-b, for BWT fluctuations as shown in Figure 11-a and for a hydrate latent heat equal to zero b) methane-ethane (99\% and $1 \%$ respectively) hydrate fields. 

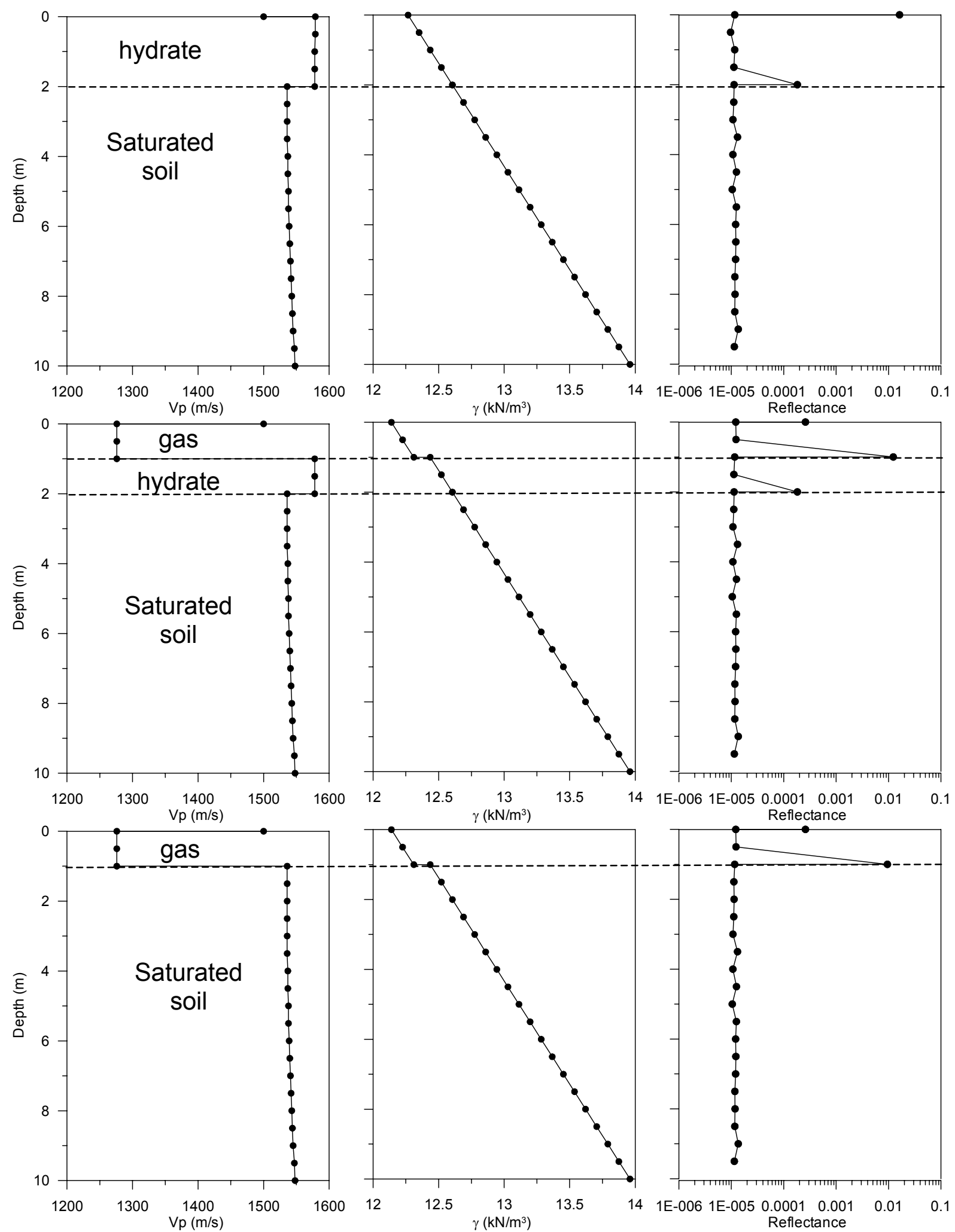

Figure 14. P-wave velocity, unit weight and reflectance versus depth determined for a) hydrate - saturated soil interface, b) gas, hydrate and saturated soil interfaces and c) gassaturated soil interface 

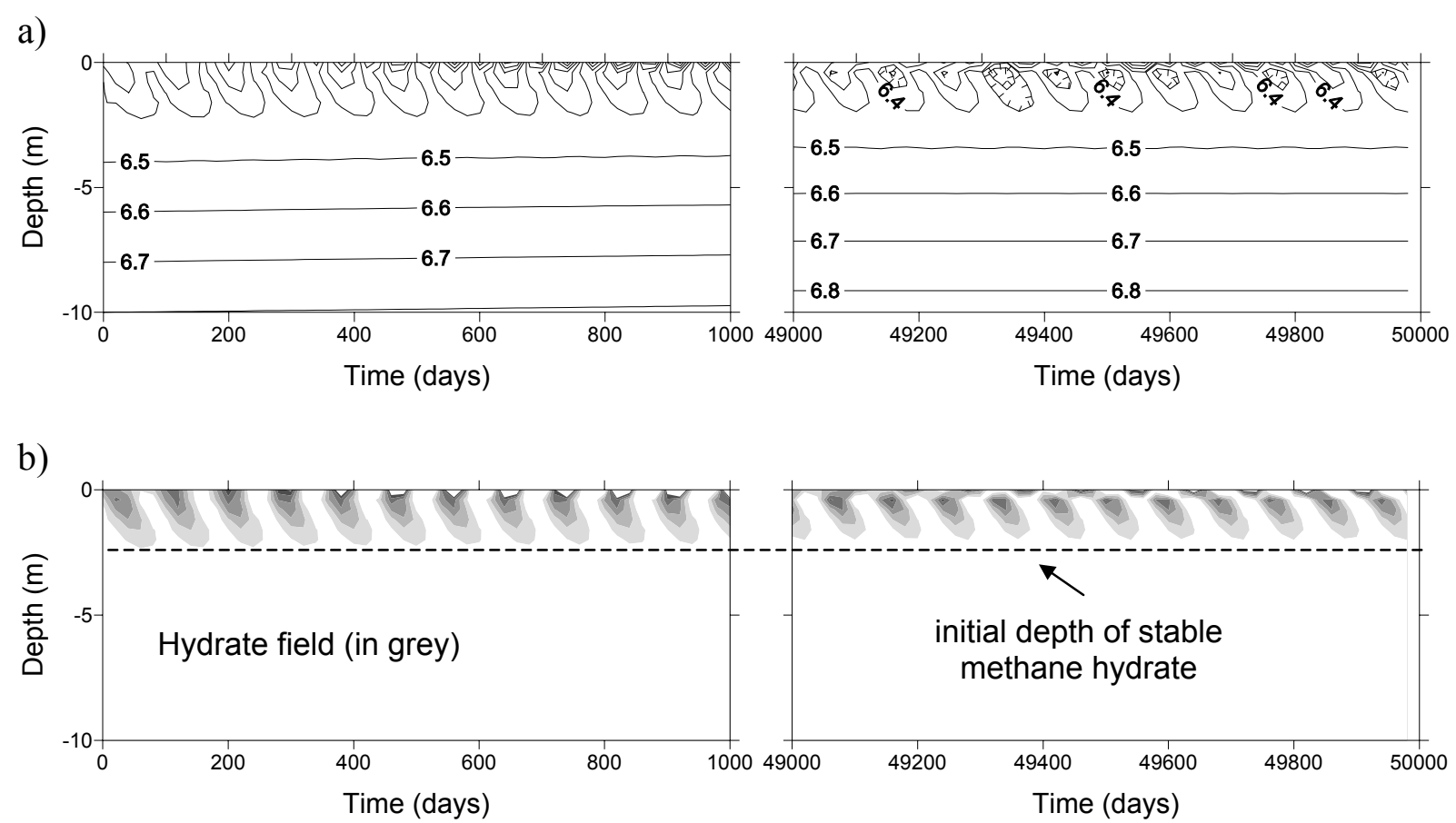

Figure-B 1. a) Temperature in the upper $10 \mathrm{~m}$ of the Congo slope, at a water depth of $557 \mathrm{~m}$, calculated for an initial temperature profile presented in Figure 11-b, for BWT fluctuations as shown in Figure 11-a and for a hydrate latent heat equal to zero b) methane hydrate fields for zero latent heat. 